\section{OPEN ACCESS}

Edited by:

Victor Ramírez-Amaya,

Medical Research Institute Mercedes and Martín Ferreyra (INIMEC),

Argentina

Reviewed by:

Chuang Wang,

Ningbo University, China

Ricardo Marcos Pautassi

Medical Research Institute Mercedes and Martín Ferreyra (INIMEC),

Argentina

*Correspondence:

Li-ping Yang

bioy/p@126.com;

Lbioylp@126.com

†These authors share first authorship

Received: 18 April 2020

Accepted: 03 August 2020

Published: 04 September 2020

Citation:

Li Y-j, Yang L-p, Hou J-I, Li X-m,

Chen L, Zhu J-h, Wang Q-y, Li G,

Zhao P-y, Liu X-h and Shi Z-j (2020)

Prenatal Stress Impairs Postnatal

Learning and Memory Development

via Disturbance of the CGMP-PKG

Pathway and Oxidative

Phosphorylation in the Hippocampus of Rats. Front. Mol. Neurosci. 13:158.

doi: 10.3389/fnmol.2020.00158

\title{
Prenatal Stress Impairs Postnatal Learning and Memory Development via Disturbance of the CGMP-PKG Pathway and Oxidative Phosphorylation in the Hippocampus of Rats
}

\begin{abstract}
Yu-jie $\mathrm{Li}^{1+}$, Li-ping Yang ${ }^{2 * t}$, Jun-lin $\mathrm{Hou}^{2}$, Xin-min $\mathrm{Li}^{2}$, Lei Chen², Jiang-hui Zhu', Qi-yang Wang ${ }^{2}$, Gai Lí2, Pei-yuan Zhao ${ }^{2}$ Xi-hong Liu' ${ }^{2}$ and Zhan-jiang Shi'
\end{abstract}

\footnotetext{
' Pharmacology Laboratory, School of Basic Medical Medicine, Henan University of Chinese Medicine, Zhengzhou, China, ${ }^{2}$ Department of Integrated Traditional Chinese and Western Medicine, School of Basic Medical Medicine, Henan University of Chinese Medicine, Zhengzhou, China
}

Clinical and animal studies have found that prenatal stress can lead to pathological changes in embryos and fetuses. However, the mechanisms through which this occurs have not been made clear. In the present study, pregnant rats were subjected to chronic psychological stress during gestational days using an improved communication box system, and the changes in behavioral performance and proteins in the hippocampus of offspring were analyzed. It was found that prenatal stress caused postnatal growth retardation and impairment in spatial learning and memory. Furthermore, in isobaric tags for relative and absolute quantitation-based proteomics analyses, 158 significantly differentially expressed proteins (DEPs) were found between the two groups. Further analyses showed that these DEPs are involved in different molecular function categories and participate in several biological processes, such as energy metabolism, learning or memory, and synaptic plasticity. Moreover, the enrichment of pathways showed that the learning and memory impairment was primarily connected with the cyclic guanosine monophosphate-protein kinase G (cGMP-PKG) pathway and oxidative phosphorylation. At the same time, the cGMP level and the expression of PKG protein were significantly decreased, and the neuronal mitochondria appeared to have a swollen and irregular shape in the hippocampus of offspring of stressed rats. These results suggest that the chronic psychological stress that pregnant rats were subjected to during gestational days may have impaired the spatial learning and memory of offspring. This affected the hippocampal oxidative phosphorylation and inhibited the cGMP-PKG pathway.

Keywords: prenatal stress, iTRAQ, learning and memory, oxidative phosphorylation, cGMP-PKG pathway 


\section{INTRODUCTION}

Clinical and animal studies have shown that prenatal stress can lead to pathological changes in embryos and fetuses. The environmental adversity that is experienced by the mother during pregnancy, whether emotional or physical, affects the growth of the fetus and the physical and mental health of the offspring (Laplante et al., 2004; Soliani et al., 2018). However, the mechanisms through which prenatal stress affects offspring are not yet clear. Many researchers have found that stress may alter the set points of the hypothalamic-pituitary-adrenal and the corticotropin-releasing factor systems and also increase the level of glucocorticoids (Ladd et al., 2000; Welberg and Seckl, 2001). The level of fetal exposure to maternal corticosterone is regulated by the high-affinity, high-efficiency type- 2 isoform of the placental enzyme 11/-hydroxysteroid dehydrogenase. However, prolonged exposure to high levels of glucocorticoids in the placenta results in the decreased activity of type- 2 isoform $11 \beta$-hydroxysteroid dehydrogenase, which leads to a relatively large increase in corticosterone that reaches fetal blood flow (Jensen Pena et al., 2012). At a behavioral level, prenatal stress can enhance emotionality and depression-like behavior, and it can affect spatial learning and memory ability, leading to anxiety-like behavior in offspring (Weinstock, 2001, 2017; Abe et al., 2007; Wang Y. et al., 2015). Prenatal stress can affect the density of the dendritic spine and the dendritic complexity in the hippocampus and the prefrontal cortex of the offspring, and some of these changes are sex specific (Schmidt et al., 2018). At the level of molecular biology, prenatal stress affects the kynurenic acid branch of the kynurenine pathway metabolism in the fetal brain. This may constitute a molecular link between prenatal stress and postnatal brain development (Notarangelo and Schwarcz, 2016). Moreover, prenatal stress-induced depressivelike behavior correlates with hippocampal Avp and Oxt receptor expression, but only in female offspring (Schmidt et al., 2018). In addition, prenatal stress that affects the behavior of offspring might relate to the maternal activation of the renin-angiotensinaldosterone system (Senko et al., 2017) or through the epigenetic inheritance mechanism of DNA methylation modifications, which may change the expression of genes and the metabolome (Youssef et al., 2018). However, previous studies have not presented a comprehensive understanding of the mechanisms of pathological changes in offspring caused by prenatal stress. A powerful combination of metabonomics and proteomics is often used in pathophysiological studies of depression and spatial memory deficiency (Palmfeldt et al., 2016; Wang et al., 2017; Zhang et al., 2018). Therefore, the results of a comprehensive analysis of metabonomics may be helpful for identifying potential biological relationships between prenatal stress and pathological changes in offspring.

Our previous research has demonstrated that psychological fear stress during pregnancy may affect the cognitive development of offspring, and the mechanism for this might be an imbalance of neurotransmitter secretion in the brain (Xinmin et al., 2017; Liping et al., 2018). However, the precise mechanism has remained unclear. In this study, we examined the hippocampal protein profiles of offspring to determine whether they were affected by prenatal fear stress. The fear stress model (FSM) of pregnant rats was prepared by observing the electrical stimulation of male rats according to the method described in previous studies (Liping et al., 2018; Geng et al., 2019). A proteomic approach based on isobaric tags for relative and absolute quantification (iTRAQ) and a metabolomic approach based on liquid chromatography mass spectrometry (LC-MS) were employed to obtain unbiased profiling data. The STRING database and Cytoscape were used to construct protein-protein interaction networks. Gene Ontology (GO) analyses were performed to analyze the main function of the differentially expressed proteins (DEPs), and the Kyoto Encyclopedia of Genes and Genomes (KEGG) was used to identify the significant pathways of these DEPs. The results help elucidate the complex molecular mechanisms of how prenatal stress can cause the neuronal damage of offspring. They may guide the creation of strategies for the diagnosis and the prevention of diseases caused by prenatal psychological stress.

\section{MATERIALS AND METHODS}

\section{Animal Care and Ethics Statement}

A total of 65 (40 female) adult Wistar rats [specific-pathogenfree grade; age, 11 weeks; weight, 170-210 g; Shandong Lu Kang Medical Limited by Share Ltd., Shandong, China; license number: SCXK (Lu) 2014-0001] were used in the present study. All the rats were housed in standard laboratory conditions $\left(21 \pm 1^{\circ} \mathrm{C}\right.$, $55 \pm 5 \%$ relative humidity, $12 / 12 \mathrm{~h}$ light/dark cycle) and had ad libitum access to food and water. The study protocol was approved by the Ethics Committee of Henan University of Chinese Medicine (Henan, China). All the animal treatments were performed according to the National Institutes of Health guidelines (Clark et al., 1997).

\section{Animal Treatment \\ Experimental Protocol}

All rats were housed for 7 days to acclimate them to the environment. Body weight and scores on the Sucrose Preference Test (SPT) (Wu et al., 2016) and the Open-Field Test (OFT) (Smart and Dobbing, 1971) were measured for the rats to ensure the consistency of the research objects and to minimize individual differences. Two outliers were screened using a box plot and were removed. The male rats were measured as well, and one outlier was restricted from mating but not removed. Later, it was used for electrical stimulation tests. When presenting proestrus or estrus, the rats were placed together in a 2:1 ratio of females to males (three rats per box). Mating and gestation were estimated by the presence of a vaginal plug. If it was not found, a vaginal smear was used to examine the presence of sperm. Mating was allowed to continue, and the rats were checked again on the following day. If no vaginal plug or sperm was found, the same procedures were followed for 3 days. After mating, the pregnant rats (those for which a vaginal plug or sperm was found) were randomly divided into the FSM group and the normal control (NC) group, with 15 rats per group. The pregnant rats from the FSM group observed the electrical stimulation of male rats for 20 
days, using an improved communication box system, according to previously described methods (Xinmin et al., 2017; Liping et al., 2018; Geng et al., 2019). After this treatment, behavioral changes in the pregnant rats (and their offspring after birth) were observed (more details below). Eventually, the offspring were sacrificed and their hippocampi were excised to examine the relationship between maternal stress during gestational days and brain injury in offspring.

The behavioral assessment of pregnant rats proceeded as follows. During modeling, all the rats were regularly fed, and the consumption of water and food in both groups was monitored daily. The pregnancies lasted for about 21 days. At the end of each gestational week, the scores in the SPT, OFT, and Tail Suspension Test (TST) (Takeuchi et al., 2003; Wu et al., 2016) were observed to evaluate the anxiety of pregnant rats.

The behavioral assessment of the offspring (Smart and Dobbing, 1971; Chiavegatto et al., 1997; Chen et al., 2012; Ivani et al., 2016) was conducted as follows. The number, the weights, and the changes in weight of the pups for each dam were recorded and analyzed statistically. The physical development and the maturation of the pups were evaluated using the methodology proposed by Smart and Dobbing (1971), Chiavegatto et al. (1997), Chen et al. (2012), and Ivani et al. (2016). First, the dates on which surface righting reflexes occurred were recorded (Ivani et al., 2016), with the test performed during postnatal days (PNDs) 421 , by gently placing the pup in a supine position and observing whether it could right itself and bring all four of its limbs into contact with the surface within $3 \mathrm{~s}$. Those that succeeded were considered to have met the standard. Next, the dates of eye opening (beginning during PNDs 10-16), incisor eruption (beginning on PND 11), and ear opening (beginning on PND 15) were noted. Then, beginning on PND 12, we assessed whether the pups could perform the auditory startle reflex (Ivani et al., 2016), showing a whole-body startle response in response to a loud clap of the hands at less than $15 \mathrm{~cm}$ away. These tests were performed until $100 \%$ of the pups reached each developmental milestone. In addition, the OFT (PND 21), the Morris Water Maze (MWM) (PND 17-21), and the TST (PND 21) were performed to evaluate the pups' anxiety, learning, and memory.

The offspring ( $n=12$ per group) were anesthetized with chloral hydrate and sacrificed by decapitation at 21 days after birth. The brain was rapidly removed, and the hippocampus was separated. A proteomic approach using $\mathrm{iTRAQ}^{\circledR}$ and a metabolomic approach using LC-MS, ELISA, and western blotting were used to measure changes in proteins in the hippocampus of each offspring to describe the complex molecular mechanisms through which prenatal stress may cause neuronal damage. Another 12 offspring were anesthetized with chloral hydrate, followed by $4 \%$ paraformaldehyde perfusion, and their hippocampi were rapidly separated and cut into $1 \times 1 \mathrm{~mm}$ pieces and immersed in glutaraldehyde to observe the structures of hippocampal neurons through electron microscopy.

\section{Open Field Test}

OFT behavior was observed following the standard protocol (Smart and Dobbing, 1971), with a few modifications. Briefly, the rats were tested for $5 \mathrm{~min}$ in an open-field apparatus that consisted of a bright square with a diameter of $100 \mathrm{~cm}$ and a height of $50 \mathrm{~cm}$, with the floor divided into 25 approximately equal sections. The following parameters were observed, using a camera located at a right angle above the open field apparatus (the apparatus was scrubbed with $75 \%$ alcohol solution between each test):

Ambulation frequency: number of floor units entered with all four feet

Rearing frequency: number of instances of standing on the hindlimbs without touching the wall

Self-grooming frequency: number of self-grooming actions performed.

\section{Morris Water Maze}

The Morris Water Maze test was performed following the standard protocol (Wang et al., 2017), with a few modifications. The water maze consisted of a circular pool of water, with a diameter of $100 \mathrm{~cm}$ and a height of $60 \mathrm{~cm}$, divided into four equal quadrants by two imaginary perpendicular lines crossing at the center of the pool. Before the experiment, the water maze was filled with tap water to a depth of $45 \mathrm{~cm}$ from the brim. The water temperature was controlled at $25-27^{\circ} \mathrm{C}$, and it was made opaque by adding potassium permanganate. A stable platform was submerged $1 \mathrm{~cm}$ below the surface of the water to allow the rats to easily escape from the water. The training for and administration of the MWM Test had two successive stages: place navigation (initial training) and spatial probe (the Space Exploration Test). During the initial training stage, the rats were trained for 4 days to assess the ability of each rat to obtain spatial information. Each rat was subjected to four trials per day. For each trial, which lasted for $2 \mathrm{~min}$, the platform was placed in the center of the north quadrant. The rat was placed into the water, facing the wall, at one of four starting points (north, south, east, and west) in a semirandom order, and it was allowed to search for the hidden platform. The rat was taken to the cage if it found the platform and stayed on it for about $5 \mathrm{~s}$. However, if the rat did not find the platform within $90 \mathrm{~s}$, it was guided to the platform and allowed to remain there for $30 \mathrm{~s}$. The time spent by the rats to reach the platform (escape latency times) was recorded. After 4 days of training, a spatial probe was administered to evaluate memory retention. The submerged platform was removed from the pool, and the rat was allowed to swim for $90 \mathrm{~s}$ in any of the four quadrants of the pool. The number of times that each rat crossed the original platform, the speed of swimming, and the swimming track were recorded with a video-tracking system (CG-400 Image Acquisition System; Institute of Materia Medica, Chinese Academy of Medical Sciences, Shanghai, China). After each trial, the rat was wiped dry and kept warm before being returned to its cage.

\section{Sucrose Preference Test}

The SPT (Wu et al., 2016) was conducted to investigate the hedonic state of the animals. During the first 2 days of the experiment, the rats were fed separately, and two bottles of $1 \%$ sucrose solution were placed in each cage. Then, the rats were fasted from solids and liquids for $12 \mathrm{~h}$. On the third day of the experiment, a bottle containing tap water was substituted 
for one of the bottles; the other bottle was still filled with $1 \%$ sucrose solution. After $1 \mathrm{~h}$, the position of the two bottles was changed to avoid the influence of habitual behavior on the experimental results. After another $1 \mathrm{~h}$, the volume of the remaining $1 \%$ sucrose solution and the tap water were measured. The SPT was measured using the following equation: sucrose preference $=$ sucrose consumed $/$ (sucrose consumed + tap water consumed).

\section{Tail Suspension Test}

The TST was performed following previously described procedures (Wu et al., 2016), with a few modifications. Briefly, the rats were suspended $50 \mathrm{~cm}$ above the floor using an adhesive tape placed approximately $4 \mathrm{~cm}$ from the tip of the tail for $6 \mathrm{~min}$. Immobility was defined as the absence of movements of the limbs or body, except for those caused by respiration, as the rats hung passively and were completely motionless. During the test, the rats were separated from each other to prevent possible visual and acoustic associations. The results were expressed as the time that the animals spent immobile during the last 4 min of the 6 min session.

\section{Equipment for and Method of Preparing the Psychological Fear Stress Model}

The pregnant rats were subjected to chronic psychological stress from gestation days 1 to 20, once per day, as described in the following procedure. The electric shock box was modified according to Liping et al. (2013). A $50 \times 60 \times 60 \mathrm{~cm}$ box made of plexiglass plates was divided into nine rooms measuring $20 \times 20 \times 50 \mathrm{~cm}$, and several holes with a diameter of $1 \mathrm{~cm}$ were distributed across the partitions of each room. Iron wires were fixed at the partitions of the six rooms at the edge of the box, and these were used to let the rats climb up and avoid electric shock. The bottom consisted of uniformly arranged stainless steel wires that could be connected to a small electrical stimulator. During the fear stress experiment, three male rats were put into the three middle rooms for electrical stimulation treatment, with one rat in each room. The pregnant rats from the FSM group were put into the six rooms along the edge, with one rat in each room. After the power was switched on, the male rats were shocked, causing them to scream, jump, and lose excretory control. While the pregnant rats from the model group could climb onto the wires fixed on the partition to avoid electric shock, they could still perceive the males being shocked through hearing, vision, and smell, causing fear (Figure 1). During the stress stimulation, a stopwatch was set for $60 \mathrm{~s}$, and after the timer was finished, the alarm bell rang continuously for $60 \mathrm{~s}$. During the bell ringing, the male rats were stimulated 10 times with instantaneous 25-35 $\mathrm{V}$, which was repeated 15 times. The pregnant rats from the NC group were also placed in the electric shock box for 30 min every day, but no electrical stimulation was performed, and all other operations were the same as those in the model group.

\section{iTRAQ-Based Proteomics Analyses \\ Protein Extraction}

A sample of the hippocampus of each animal was added to liquid nitrogen to be ground into powder and mixed well

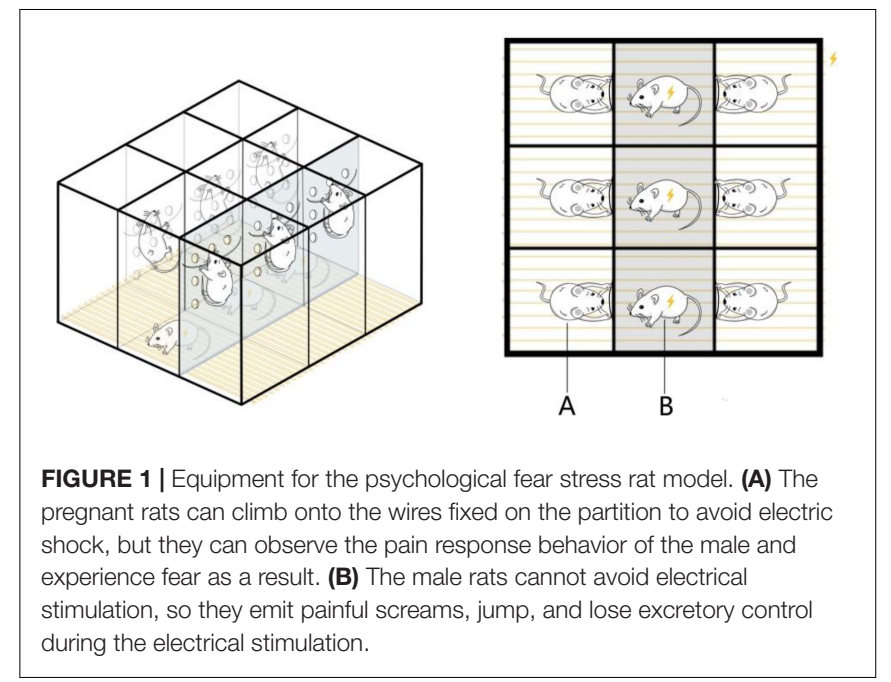

with $6 \times$ volume of chilled trichloroacetic acid (TCA)-acetone $\left(500 \mathrm{ml}\right.$ acetone, $50 \mathrm{~g} \mathrm{TCA}$ ) and incubated at $-20^{\circ} \mathrm{C}$ for at least $2 \mathrm{~h}$. After centrifugation at $4^{\circ} \mathrm{C}$ and $20,000 \times g$ for $30 \mathrm{~min}$, the supernatant was discarded, and the precipitate was dissolved with lysis buffer ( $8 \mathrm{M}$ urea, $30 \mathrm{mM}$ 4-(2-hydroxyethyl)1-piperazineethanesulfonic acid, $1 \mathrm{mM}$ phenylmethylsulfonyl fluoride, $2 \mathrm{mM}$ ethylenediaminetetraacetic acid, and $10 \mathrm{mM}$ dithiothreitol) and sonicated at $180 \mathrm{~W} 30$ times (pulse on for $2 \mathrm{~s}$ and pulse off for $3 \mathrm{~s}$ for each time). After being centrifuged at $4^{\circ} \mathrm{C}$ and $20,000 \times g$ for $30 \mathrm{~min}$, the supernatant was collected and incubated at $56^{\circ} \mathrm{C}$ for $1 \mathrm{~h}$, then added to iodoacetamide $(55 \mathrm{mM})$, and incubated in the dark for $1 \mathrm{~h}$ and subsequently mixed well with $4 \times$ volume of chilled TCA-acetone before being incubated at $-20^{\circ} \mathrm{C}$ for at least $3 \mathrm{~h}$. After centrifugation at $4^{\circ} \mathrm{C}$ and $20,000 \times g$ for $20 \mathrm{~min}$, the supernatant was discarded, and the precipitate was redissolved in $300 \mu \mathrm{l}$ dissolution buffer [50\% tetraethylammonium bromide (TEAB) and $0.1 \%$ sodium dodecyl sulfate (SDS)] and sonicated at $180 \mathrm{~W}$ for $3 \mathrm{~min}$. The protein concentrations were quantified using the Bradford method [Pierce Coomassie (Bradford) Protein Assay Kit, Thermo Fisher Fisher].

\section{Protein Processing and iTRAQ Labeling}

Total protein $(100 \mu \mathrm{g})$ from each sample was added to the dissolution buffer (50\% TEAB and 0.1\% SDS). Then, $3.3 \mu \mathrm{g}$ trypsin was added to each sample, and the samples were incubated at $37^{\circ} \mathrm{C}$ for $24 \mathrm{~h}$. This was repeated with $1 \mu \mathrm{g}$ trypsin incubated at $37^{\circ} \mathrm{C}$ for $12 \mathrm{~h}$ to digest the protein. Subsequently, the peptide segment was freeze-dried and redissolved with 30 $\mu \mathrm{l}$ dissolution buffer (50\% TEAB and $0.1 \%$ SDS). The digestion efficiency was quantified via MALDI Tof/Tof. The sample was labeled with iTRAQ reagent, as follows. The tryptic peptides were labeled with an iTRAQReagent-8Plex Multiplex Kit (Applied Biosystems), following the manufacturer's protocol. The $\mathrm{NC}$ group was labeled with iTRAQ reagent 119, and the FSM group was labeled with iTRAQ reagent 121. Incubation was allowed to proceed at room temperature for $2 \mathrm{~h}$ and then was stopped 
by adding $10 \mathrm{mM} \mathrm{KH} \mathrm{PO}_{4}$ and $25 \%$ acetonitrile (ACN) at $\mathrm{pH}=3.0$. Subsequently, the two labeled samples were pooled and preliminarily separated using strong cation exchange (SCX) high-performance liquid chromatography. Briefly, the peptides were dissolved and loaded onto an SCX chromatographic column (Luna SCX $250 \times 4.60 \mathrm{~mm} 100 \AA$, phenomenex) in buffer $\mathrm{A}$ $\left(10 \mathrm{mM} \mathrm{KH} \mathrm{PO}_{4}\right.$ in $\left.25 \% \mathrm{ACN}, \mathrm{pH}=3.0\right)$ and equilibrated in $100 \%$ solution $\mathrm{A}$ for $10 \mathrm{~min}$, followed by fast elution in a gradient of $0-10 \% \mathrm{~B}\left(2 \mathrm{M} \mathrm{KCL}\right.$ and $10 \mathrm{mM} \mathrm{KH}_{2} \mathrm{PO}_{4}$ in $25 \%$ $\mathrm{ACN}, \mathrm{pH}=3.0$, flow rate $=1 \mathrm{ml} / \mathrm{min}$ ) for $5 \mathrm{~min}, 10-20 \%$ B for $20 \mathrm{~min}, 20-30 \% \mathrm{~B}$ for $5 \mathrm{~min}$, and $30-50 \%$ solution $\mathrm{B}$ for $3 \mathrm{~min}$. The fractioned peptides were collected at a rate of one tube per minute during the elution period. Each fraction was desalted using a C18 column (strata-X C18, phenomenex), lyophilized, and dissolved in $0.1 \%$ formic acid for LC-MS/MS analyses (Chen et al., 2016).

\section{LC-MS/MS Analyses}

The peptides in each fraction were delivered into EASY-nLC (PROXEON) and eluted with 5-45\% ACN in $0.1 \%$ formic acid for $90 \mathrm{~min}$ at $300 \mathrm{nl} / \mathrm{min}$. The peptides eluted from LC were analyzed using a hybrid quadrupole/time-of-flight mass spectrometer (microTOF-Q II, Bruker) (Chen et al., 2016). The source parameters were as follows: capillary voltage, 1,350 V; dry gas, $3.0 \mathrm{~L} / \mathrm{min}$; and dry temperature, $150^{\circ} \mathrm{C}$. The acquisition range was $300-1,500 \mathrm{~m} / \mathrm{z}$ for the MS and $50-3,000 \mathrm{~m} / \mathrm{z}$ for the MS/MS. The absolute threshold for both MS and MS/MS was 1,000 intensity counts. The spectra time for MS was set to $0.5 \mathrm{~s}$, that for MS/MS with 1,000-10,000 intensity counts was set to 1.2 s, and that for MS/MS with $>10,000$ intensity counts was set to $0.5 \mathrm{~s}$. The auto MS/MS mode was used with positive ion polarity, and the preferred range of the charge state was set from $2+$ to $5+$, with no single charge. The collision energy for fragmentation was set to certain dynamic ranges for different charge states: $12-$ $72 \mathrm{eV}$ for $1+, 22-62 \mathrm{eV}$ for $2+, 17-57 \mathrm{eV}$ for $3+, 15-55 \mathrm{eV}$ for $4+$, and $12-52 \mathrm{eV}$ for $5+$. Collision energy sweeping was set as follows: start 100\% (timing 55\%) and end 100\% (timing $45 \%)$. Three precursor ions were collected per cycle with active exclusion (1 $\mathrm{min})$.

\section{Data Analyses}

Raw data files were converted into MGF files using Proteome Discoverer 1.3 (Thermo Fisher Scientific). The proteins were identified using Mascot 2.3.0 (Matrix Science, Boston, MA, United States), based on the ipi-rat-v3-85-1 database and quantified using Proteome Discoverer 1.3. The user-defined search parameters were as follows: fixed modification to carbamidomethyl (C), variable modification to oxidation (M), Gln-Pyro-Glu to N-term Q, iTRAQ 8-plex to K, iTRAQ 8-plex to Y, iTRAQ 8-plex to N-term, enzyme to trypsin, peptide tolerance to $0.1 \mathrm{Da}, \mathrm{MS} / \mathrm{MS}$ tolerance to $0.1 \mathrm{Da}$, and maximum missed cleavages to 1 . The quantitative analysis parameters were set as follows: protein ratio type to median, minimum peptides to 1, peptide threshold type to at least homology, normalization method to median, $P$-value to $<0.05$, peptide false discovery rate to $<1 \%$, and iTRAQ reporter ratio change to $>1.2$-fold or $<0.83$-fold.

\section{Bioinformatics Analysis}

The STRING database and Cytoscape were used for the construction of protein-protein interaction networks. GO analyses were performed to analyze the main functions of the DEPs, and KEGG (Kanehisa Laboratories, Kyoto, Japan) was used to identify the significant pathways of the DEPs. The Cytoscape ClueGO software tool was used to perform GO analyses and pathway analyses (Bindea et al., 2009; Liu et al., 2019; Xu et al., 2019). The parameters of the analyses were as follows: hyper-geometric distribution, two-sided (enrichment/depletion) tests, statistical significance of $p \leq 0.05$, correction with the Bonferroni adjustment, a Kappa-statistic score of 0.4, GO-level intervals of 3-8, leading group at highest significance, and group merge of $50 \%$.

\section{Western Blot}

The hippocampus samples were lysed in RIPA Lysis Buffer (Beyotime Biotechnology, Shanghai, China, catalog P0013B). The lysates were incubated on ice for $10 \mathrm{~min}$ and centrifuged at $4^{\circ} \mathrm{C}$ and $12,000 \times g$ for $5 \mathrm{~min}$, and then the supernatants were collected. The protein concentrations were quantified using a bicinchoninic acid kit (Tiangen, China). The protein lysates were separated by SDS- polyacrylamide gel electrophoresis (4-6\%) and transferred to a polyvinylidene fluoride membrane (Millipore, Schwalbach, Germany). After blocking with 5\% skimmed milk in Tris-buffered saline Tween-20 at room temperature for $1 \mathrm{~h}$, the membranes were incubated with the following primary antibodies against PKG (CST, catalog 3248, Boston, MA, United States) in Tris-buffered saline Tween-20 at $4^{\circ} \mathrm{C}$ overnight. After washing, the membranes were incubated in horseradish peroxidase-conjugated secondary antibody $(1: 1,000)$ at room temperature for $1 \mathrm{~h}$. The bound antibodies on the membranes were visualized using enhanced chemiluminescence. With glyceraldehyde 3-phosphate dehydrogenase, the antibody was used as a control for protein loading. The relative level of each protein to the control protein was quantified using Image-Pro Plus 6.0 software (Media Cybernetics, Rockville, MD, United States).

\section{Electron Microscopy}

Transmission electron microscope images were prepared by the Electron Microscope Center of Scientific Research and Experiment Center of Henan University of Chinese Medicine. Tissue pieces of the hippocampus were fixed with $2.5 \%$ glutaraldehyde for $4 \mathrm{~h}$ and washed four times with 0.1 $\mathrm{M}$ phosphate-buffered saline (PBS) for $15 \mathrm{~min}$ each time. After rinsing, the samples were placed into $1 \%$ osmium acid fixative solution and fixed again for $1.5 \mathrm{~h}$. Then, the samples were rinsed with $0.1 \mathrm{M}$ PBS for $15 \mathrm{~min}$ each time. The tissues were dehydrated with graded alcohol series (50, 70, 80, and 100\%) and embedded in a mixture of epoxy resin 812 and acetone (1:1), epoxy resin 812 and acetone (2:1), and pure epoxy resin 812, respectively, and placed overnight at room temperature. Then, these were sliced into serial coronal 50-60 nm thick sections using an ultrathin slicing machine and dyed in saturated uranium dioxide 
acetate solution for $20 \mathrm{~min}$. After rinsing and drying, the sections were observed and photographed by transmission electron microscopy.

\section{Morphometric Analysis of Mitochondria}

Morphometric analyses of mitochondria were performed with the Fiji Software on a sample of 10 systematically, uniformly, and randomly selected images (Paukov et al., 1971). First, the mean number of mitochondria per electron micrograph was calculated. The second index of the state of the mitochondria ultrastructure was the mean number of cristae per mitochondrion. The third index of the state of the mitochondria ultrastructure was the area of the mitochondria, directly reflecting the volume of these organelles. The fourth index of the state of the mitochondria ultrastructure was the coefficient of energy efficiency of mitochondria (CEEM). This coefficient is the product of the number of mitochondrial cristae and the area of mitochondria.

\section{Statistical Analyses}

Measurement data are presented as means \pm SDs, and the statistical analyses were performed using SPSS 21.0 statistical software (IBM, Armonk, NY, United States). The scores for the SPT, TST, and OFT for pregnant rats and the escape latency of offspring were analyzed using repeated-measures ANOVA. An independent $t$-test was conducted to analyze the body weights of the pregnant rats and the birth weights and scores of the offspring. Two-way ANOVA was conducted to analyze the TST, OFT, and Space Exploration Test of the offspring. $P<0.05$ was considered as statistically significant.

\section{RESULTS}

\section{Weight Changes in Pregnant Rats During Gestation}

Because some females were not pregnant after the mating period, five rats were excluded from the NC group and three from the
FSM group, leaving 10 rats in the NC group and 12 rats in the FSM group. As shown in Figure 2A, the dams in the NC and FSM groups had similar body weights before exposure to stress. However, after treatment, the females in the FSM group had lower body weights. On day 21 of pregnancy, their body weights were significantly less than those of the NC group $(t=6.251, P<0.01)$.

\section{Behavioral Assessment of Pregnant Rats}

Repeated-measures ANOVA showed that stimulation time had a significant effect on sucrose consumption $(F=12.535, P<0.001)$. An interaction was found between stimulation time and group $(F=32.433, P<0.001)$, and the sucrose consumption of the FSM rats was significantly less than that of the NC rats $(P<0.001)$ (Figure 2A). For the TST results (Figure 2A), a repeatedmeasures ANOVA showed that there were no differences between the groups $(P>0.05)$ and no effects of stimulation time $(F=1.264, P>0.05)$. In addition, for the OFT results (Figure $2 \mathbf{B}$ ), stimulation time had a significant effect on the frequency of ambulation $(F=5.684, P<0.01)$, rearing $(F=19.538, P<0.001)$, and self-grooming $(F=7.123, P<0.01)$. There were significant differences between the groups in terms of ambulation $(P<0.01)$ and rearing $(P<0.01)$. These results suggest that the exploratory behavior of rats in the FSM group was decreased.

\section{Physical and Reflex Development of the Offspring Rats}

The birth weights of the offspring of the FSM group (OFSM, $7.33 \pm 0.80$ ) were significantly lower than those of the offspring of the NC group (ONC, 6.25 $\pm 0.97, t=9.628, P<0.01$, Figure 3A). This suggests that fear stress in pregnant rats affected the birth weights of their offspring. Among indicators of the physical and the reflex development of the offspring, the eye opening $(t=-2.720, P<0.05)$ and the surface righting reflex $(t=-2.694, P<0.05$, Figure 3B) times of the pups in the OFSM group were significantly delayed compared to those of the ONC group. No significant differences were observed in ear opening, incisor eruption, or auditory startle reflex between the two groups $(P>0.05$, Figure 3B). These results suggest that fear stress
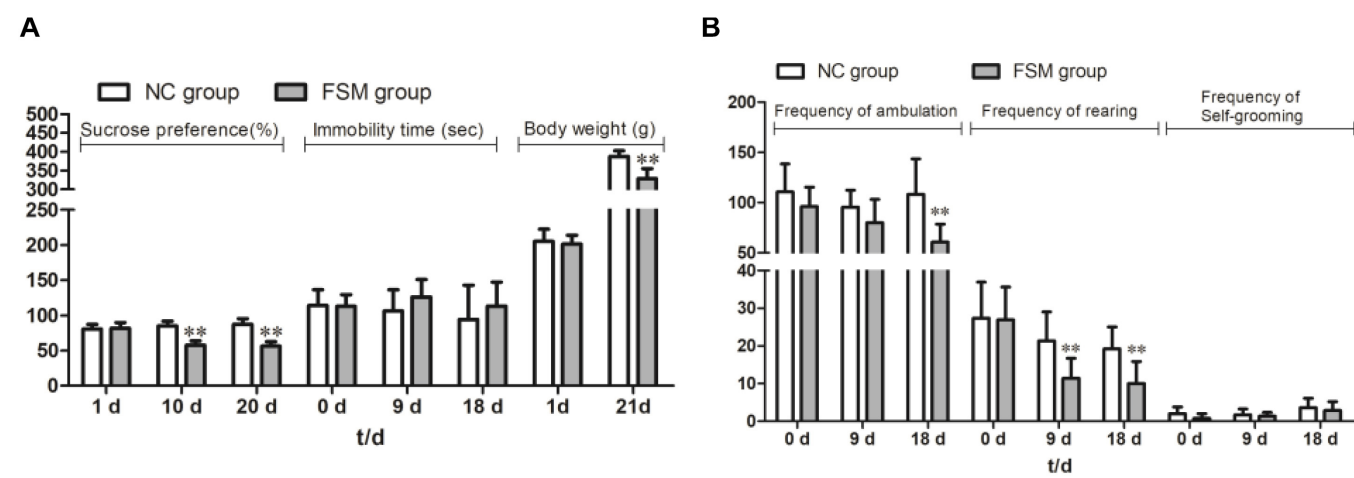

FIGURE 2 | Behavioral assessment of pregnant rats. The pregnant rats' results for (A) the Sucrose Preference Test, immobility time, and body weight changes and (B) the Open Field Test are presented. Values are expressed as means \pm standard deviations. NC group, normal control group $(n=10)$; FSM group, fear stress model group $(n=12) .{ }^{* *} P<0.01$ versus the NC group. 

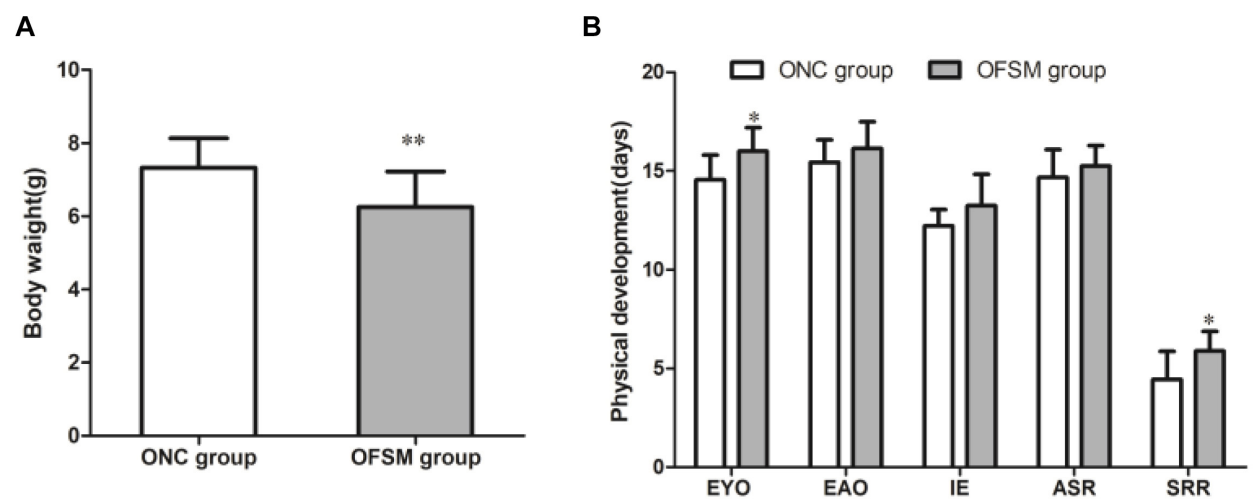

FIGURE 3 | Fear stress in pregnant rats during gestational days may affect the physical and the reflex development of offspring. (A) Birth weights of offspring. ONC group $(n=125)$ and OFSM group $(n=129)$. (B) Physical and reflex development of offspring. OFSM, offspring from the FSM group ( $n=12)$; ONC, offspring from the normal control group ( $n=10)$; EYO, eye opening; EAO, ear opening; IE, incisor eruption; ASR, auditory startle reflex; SRR, surface righting reflex. Values are expressed as means \pm standard deviations. ${ }^{\star} P<0.05$ and ${ }^{\star \star} P<0.01$ versus the ONC group.

A

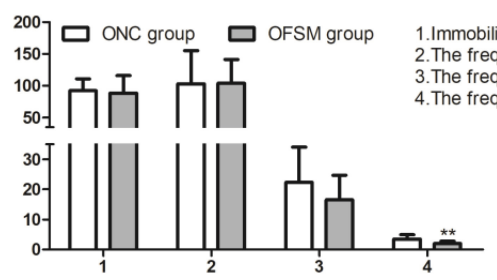

C

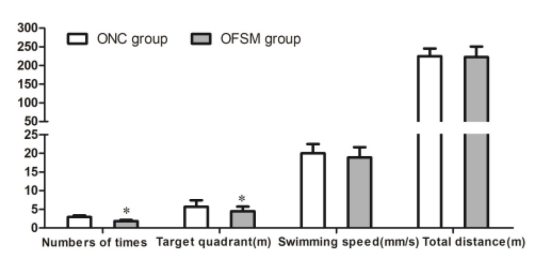

B

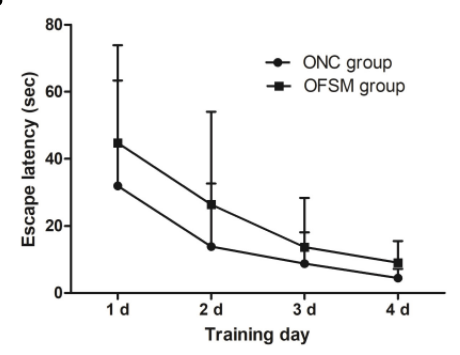

D

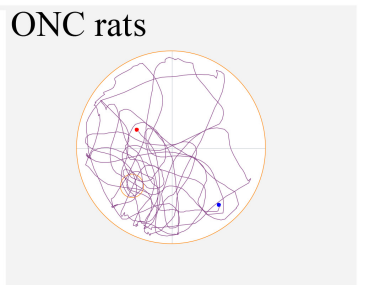

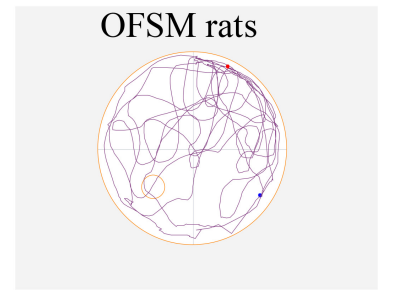

FIGURE 4 | Fear stress in pregnant rats may affect the behavior of their offspring. (A) Immobility times in the TST and the frequency of ambulation, rearing, and self-grooming in the OFT. (B) Escape latency during the four training days in the MWM. (C) Swimming speeds and distances during the probe test in the MWM. (D) Representative MWM-generated swimming tracing pattern for offspring from the normal control group (ONC) and for offspring from the fear stress model (OFSM) group in the probe tests. TST, Tail Suspension Test; OFT, Open Field Test; MWM, Morris Water Maze. Values are expressed as means \pm standard deviations. ONC group $(n=19)$, OFSM group $(n=18) .{ }^{\star} P<0.05,{ }^{\star \star} P<0.01$ versus ONC group.

may have also led to a delay in the physiological and the reflex development of offspring.

\section{Behavioral Assessment of Offspring}

To observe the psychological status of offspring, behavioral tests, including the TST, OFT, and MWM Test, were conducted on PND 21. In the TST, there were no significant differences in immobility time between the two offspring groups $(F=0.445$, $P>0.05$, Figure 4A). In addition, in the OFT scores (Figure 4A), there were no significant differences in the frequency of ambulation $(F=0.003, P>0.05)$ or frequency of rearing $(F=1.601, P>0.05)$ between the two offspring groups. However, the frequency of self-grooming was significantly lower in OFSM rats $(F=9.924, P<0.01$, Figure 4D), and there was no significant difference between different genders $(P>0.05)$. Learning occurred in both the ONC and the OFSM groups as the escape latencies became progressively shorter, but the escape latencies for the OFSM rats were longer than those of ONC rats. A repeated-measures ANOVA showed that the differences between different groups $(F=3.55, P=0.068$, Figure 4B) and genders $(F=0.09, P=0.766)$ were not statistically significant. The results of the probe test for OFSM are shown in Figure 4C. There were no significant differences in swimming speed $(F=0.105$, $P>0.05$, Figure 4C) and swimming distance $(F=1.665, P>0.05$, Figure 4C) between the two groups. The swimming distance in the target quadrant $(F=6.03, P<0.05)$ and the number 
of times that the rats crossed the original platform $(F=4.413$, $P<0.05)$ of the OFSM group significantly decreased relative to the values for the ONC rats (Figure 4C). There was no significant difference between different genders $(P>0.05)$. Overall, these results indicate that fear stress in pregnant rats might impair the spatial learning and the memory ability of their offspring. Representative tracks of the probe test are shown in Figure 4D.

\section{iTRAQ-Based Proteomic Analyses}

Next, we investigated the effects of chronic fear stress in pregnant rats on the expression of proteins in the hippocampi of their pups using iTRAQ-based proteomics analyses. Matching to the ipi-ratv3-85-1 database, within the two groups, a total of 987 proteins that contained at least one unique peptide were identified, and $0.2 \%$ false discovery rate was used for multivariate analyses. iTRAQ reporter ratios that changed $>1.2$-fold or $<0.83$-fold and $P<0.05$ versus the values for the NC group were deemed to be DEPs. Overall, 158 DEPs were found between the two groups. Of these, 58 proteins were upregulated (Table 1) and 100 were downregulated (Table 2) in the OFSM group, relative to the ONC group, that is, the number of downregulated proteins was much higher than the number of upregulated proteins.

\section{GO and KEGG Enrichment Analyses and Protein-Protein Interaction Network of DEPs}

To better understand the mechanisms of the effects of mental stress in pregnant rats on the learning and memory of their offspring, GO classification and pathway enrichment of the DEPs were performed. The results suggested that fear stress during pregnancy affected offspring metabolism, biosynthetic function, transport function, learning, and memory, among other impacts. According to GO classification, the DEPs were involved in osteoclast differentiation (30316), mitochondrial membrane organization (7006), regulation of axon regeneration (48679), regulation of transmembrane transporter activity (22898), myofibril assembly (30239), protein localization to cell surface (34394), substantia nigra development (21762), peptidyl-cysteine modification (18198), proton transport (15992), learning or memory (7611), ATP metabolism (46034), and other biological processes (Figure 5A). The DEPs were mainly enriched in the ATP metabolic process and learning or memory (Figure 5B). According to the GO cellular component annotations, the DEPs were mainly located in focal adhesion (0005925), proton-transporting two-sector ATPase complex (16469), and actin-based cell projection (98858) (Figure 5C). The GO molecular function annotations showed that the DEPs were involved in ATPase activity, coupled to the transmembrane movement of ions, rotational mechanisms (0044769), pyruvate dehydrogenase $[\mathrm{NAD}(\mathrm{P})+]$ activity (0034603), oxidoreductase activity, acting on $\mathrm{NAD}(\mathrm{P}) \mathrm{H}$ (0016651), and protein serine/threonine kinase inhibitor activity (0030291) (Figure 6B). In pathway enrichment analyses, the DEPs were related to the pentose phosphate pathway (00030), proteasome (03050), the cyclic guanosine monophosphateprotein kinase G (cGMP-PKG) signaling pathway (04022), vasopressin-regulated water reabsorption (04962), amoebiasis (05146), glycolysis/gluconeogenesis (00010), hippo signaling pathway (04390), oxidative phosphorylation (00190), and Alzheimer's disease (05010) (Figure 6A). According to pathway enrichment analyses, the DEPs were mainly enriched in Alzheimer's disease, oxidative phosphorylation, oocyte meiosis (04114, or the hippo signaling pathway, 04390), and the glycolysis/gluconeogenesis pathways (00010) (Figure 6C). The protein-protein interaction network of these proteins is shown in Figure 7. Analyses of this network revealed that ATP5C1, VDAC1, ACTB, GAPDH, and PARK7 participated in a range of biological processes. ATP5C1 interacts with multiple proteins (ATP5C1, ATP5H, ATP5F1, DLD, ATP6V1F, ATP6V1E1, and NDUFS1) that participate in oxidative phosphorylation and the ATP metabolic process. VDAC1 interacts with YWHAE, APP, PPP3R1, YWHAB, CASP1, VDAC1, SFN, NDUFS1, DYNLL1, and GAPDH, which participate in cell death. PARK7 interacts with multiple proteins, which participate in behavior, oxygen and reactive oxygen species metabolism, regulation of neurotransmitter levels, and synaptic transmission. These changes may play an important role in the impairment of learning and memory function in offspring.

\section{Effects of Chronic Fear Stress in Pregnant Rats on the cGMP/PKG Pathway in the Hippocampus of Offspring}

Pathway enrichment analyses show that a rather high number of DEPs are related to the Alzheimer's disease pathway. As shown in Figure 6A, a disturbance in the cGMP-PKG signaling pathway of the hippocampus was involved in learning and memory impairment. Thus, the cGMP level and the expression of PKG protein in the hippocampus were further analyzed using ELISA and western blotting. The results are shown in Figure 8. We found that the cGMP level and the PKG protein expression were significantly lower in the hippocampus of rats in the OFSM group than in those of the ONC group $(P<0.01)$. These results suggest that prenatal stress inhibited the $\mathrm{CGMP} / \mathrm{PKG}$ signaling pathway, which led to memory impairment in postnatal rats.

\section{Effects of Chronic Fear Stress in Pregnant Rats on the Ultrastructure of Neurons in the Hippocampus of Offspring}

As shown in Figure 6, according to the pathway enrichment and network analyses, high numbers of DEPs were related to oxidative phosphorylation, which is involved in learning and memory impairment. Oxidative phosphorylation in the mitochondria is the main site for the production of ATP in neuronal cells, so the ultrastructure of the neurons in the hippocampus of the offspring was observed with electron microscopy. The results are shown in Figure 8D. The neuronal shape, nuclear membrane, and nucleolus were clearly visible in the ONC group, chromatin was distributed relatively evenly, the organelle structure was normal, the mitochondrial membrane and the mitochondrial 
TABLE 1 | Upregulated proteins in the hippocampus of pups.

\begin{tabular}{|c|c|}
\hline Accession & Protein names \\
\hline Q04940 & Neurogranin (Nrgn) \\
\hline D3ZTW5 & Solute carrier family 25 , member 54 (Slc25a54) \\
\hline P34926 & Microtubule-associated protein 1A (Map1a) \\
\hline Q6IMY8 & Heterogeneous nuclear ribonucleoprotein U (Hnrnpu) \\
\hline Q6P799 & Seryl-tRNA synthetase (Sars) \\
\hline P09456 & Protein kinase cAMP-dependent type 1 regulatory subunit alpha (Prkar1a) \\
\hline D3ZXP3 & H2A histone family, member X (H2afx) \\
\hline O35987 & NSFL1 cofactor (Nsfl1c) \\
\hline P60711 & Actin, beta (Actb) (fragment) \\
\hline 088201 & C-type lectin domain family 11 , member A (Clec11a) \\
\hline Q5FVQ4 & Malectin (Mlec) \\
\hline MOR5M3 & RGD1562660 (RGD1562660) \\
\hline Q62658 & FK506 binding protein 1a (Fkbp1a) \\
\hline Q6PDU1 & Serine/arginine-rich splicing factor 2 (Srsf2) \\
\hline P27139 & Carbonic anhydrase 2 (Car2) \\
\hline P04550 & Parathymosin (Ptms) \\
\hline Q56R17 & Karyopherin subunit alpha 4 (Kpna4) \\
\hline P0C5X8 & Tweety family member 1 (Ttyh1) \\
\hline Q7TNA8 & Lactate dehydrogenase A-like 6B (Ldhal6b) \\
\hline P52296 & Karyopherin subunit beta 1 (Kpnb1) \\
\hline F1M4G6 & ENSRNOG00000023547 uncharacterized protein (fragment) \\
\hline P47987 & Plasmolipin (Pllp) \\
\hline Q6P9T8 & Tubulin, beta 4B class IVb (Tubb4b) \\
\hline P28480 & T-complex 1 (Tcp1) \\
\hline Q3MHS9 & Chaperonin containing TCP1 subunit 6A (Cct6a) \\
\hline Q9JHL4 & Drebrin-like (Dbnl) \\
\hline O88767 & Parkinsonism-associated deglycase (Park7) \\
\hline Q4V7A0 & WD repeat domain 61 (Wdr61) \\
\hline 008589 & FXYD domain-containing ion transport regulator 1 (Fxyd1) \\
\hline Q9EPR9 & Phosphodiesterase 1A (Pde1a) \\
\hline Q3ZBA0 & Tectonin beta-propeller repeat containing 1 (Tecpr1) \\
\hline R9PXY3 & Phospholipase C beta 1 (Plcb1) \\
\hline Q5XIM7 & Lysyl-tRNA synthetase (Kars) \\
\hline P85972 & Vinculin (Vcl) \\
\hline P04797 & Glyceraldehyde-3-phosphate dehydrogenase (Gapdh) \\
\hline MOR5U4 & Adenylate cyclase 9 (Adcy9) \\
\hline P02688 & Myelin basic protein (Mbp) \\
\hline A0A0G2JSZ5 & Protein disulfide isomerase family $\mathrm{A}$, member 6 (Pdia6) \\
\hline Q32PX7 & Far upstream element binding protein 1 (Fubp1) \\
\hline Q2QC85 & Far upstream element binding protein 3 (Fubp3) \\
\hline
\end{tabular}


TABLE 1 | Continued

\begin{tabular}{|c|c|}
\hline Accession & Protein names \\
\hline Q5U2Z3 & Nucleosome assembly protein 1-like 4 (Nap1|4) \\
\hline Q9Z2L0 & Voltage-dependent anion channel 1 (Vdac1) \\
\hline Q63014 & A-kinase anchoring protein 8 (Akap8) \\
\hline Q03346 & Peptidase, mitochondrial processing beta subunit (Pmpcb) \\
\hline Q51017 & Similar to T cell receptor V-alpha J-alpha (LOC290071) \\
\hline
\end{tabular}

cristae were distinct, the complete outline of the synaptic membrane and the synaptic cleft was clear, and a large amount of bright round vesicles was seen distributed in the presynaptic terminals, while the postsynaptic density was thick or long in the hippocampal region. In the OFSM group, the synaptic structure was abnormal, the membranes of pre-axoaxonic synapses and post-axoaxonic synapses were not clear, the synaptic space disappeared, and the number of synapse vesicle was decreased. The neuronal mitochondria appeared swollen and vacuolated, and their cristae were fragmented (Figures $\mathbf{8 D}-\mathbf{F}$ ). The rough endoplasmic reticulum and Golgi apparatus were distended, and the membrane-bound ribosomes were denuded. In addition, a slight deformity of the nucleus and an enlargement of the perinuclear space were observed.

A morphometric analysis revealed that the total numerical density of damaged mitochondria in the hippocampus of the OFSM group was $25.96 \%$ higher than that of the ONC group (Figure 8E). A further calculation of hippocampal CEEM revealed that energy production by the hippocampal mitochondria was reduced by $28.47 \%$ in OFSM rats compared to ONC rats (Figure 8E).

\section{DISCUSSION}

We investigated the effects of chronic fear stress in pregnant rats on the expression of proteins in the hippocampus of pups using iTRAQ-based proteomics analyses, which could help improve the health and the well-being of mothers and children. We found that chronic fear stress during pregnancy had a significant impact on the growth, development, and cognitive function of offspring. Similar results have been reported by Guan et al. (2016), who found that long-term maternal stress may destroy the spatial learning and memory abilities of offspring. The mechanism was determined to be an increase in corticosterone levels in maternal plasma and a decrease in hippocampal brain-derived neurotrophic factor and Arc in offspring. However, this study validated only some specific proteins and neurotrophic factors, and it remains poorly understood how chronic fear stress during pregnancy affects other proteins in offspring. The hippocampus plays an important role in early memory formation (Martin et al., 2008). Proteomics studies should be pursued to address the effects of prenatal stress on protein expression in the hippocampus of postnatal rats. In this study, pregnant rats were exposed to chronic fear stress during gestational days through observation of the electrical stimulation of male rats. iTRAQ-based proteomic and LC-MS-based metabolomic approaches and western blotting were used to elucidate changes in proteins in the hippocampus of offspring to understand the interface between maternal stress during gestational days and injury of offspring. The results showed that the DEPs that are most centrally involved affect energy metabolism and learning or memory. These findings may lead to new insights into the mechanisms of maternal stress during gestational days, leading to the development of depression and poor spatial learning and memory abilities of offspring.

Psychological stress is a common and important cause of premature ovarian failure. This is largely due to the means through which stress affects the function of the hypothalamicpituitary-target gland axis, which leads to disorders of the hypothalamus-pituitary-ovary axis and causes premature ovarian failure (Wang X. F. et al., 2015). In addition, the Emotion-Caused Disease Theory is an important part of the etiology and the pathogenesis of the traditional system of Chinese medicine. In this study, an electric shock box was modified according to the communication box system. In this device, after the power was switched on, the male rats were shocked, causing them to scream, jump, and lose excretory control. The pregnant rats could climb up to the wires fixed on a partition to avoid the shock, but they could still hear, see, and smell what was happening to the males, which caused stress. The main advantage of this model was that it was an exclusively psychological stress model as the stimulation itself had no serious physiological effects on the pregnant rats, such as hunger, pain, or other negative stimuli, which could affect the outcome of the fetus. The commonly used animal models for chronic psychological stress include the chronic unpredictable mild stress (Zhang et al., 2018), social disruption stressor (Doherty et al., 2018), and social conflict stress (Partrick et al., 2018) models, all of which include combinations of psychological and physiological stimulation. However, diverse stimulation modes have had different effects on animals, including psychological, behavioral, learning, memory, neuroendocrine, and neurochemical effects (Katsura et al., 2002; Haleem et al., 2014). Thus, the psychological stress model in our study was ideal for a maternal stress assay. In addition, to prove the scientific reliability and rationality of the model, we evaluated it in terms of weight gain and behavior performance. The results showed that the body weight, sucrose preference, and exploratory behavior of the pregnant rats subjected to fear stress for 21 days were significantly decreased. The pregnant rats showed an apparent state of depression (Junlin et al., 2018). In previous studies, we concluded that maternal stress during gestational days resulted in behavioral changes and growth retardation in offspring. To confirm the repeatability and the reproducibility of the method and ensure experimental precision, we closely evaluated the growth and the development of offspring and their behavior. The results for birth weight, physical and reflex 
TABLE 2 | Downregulated proteins in the hippocampus of pups.

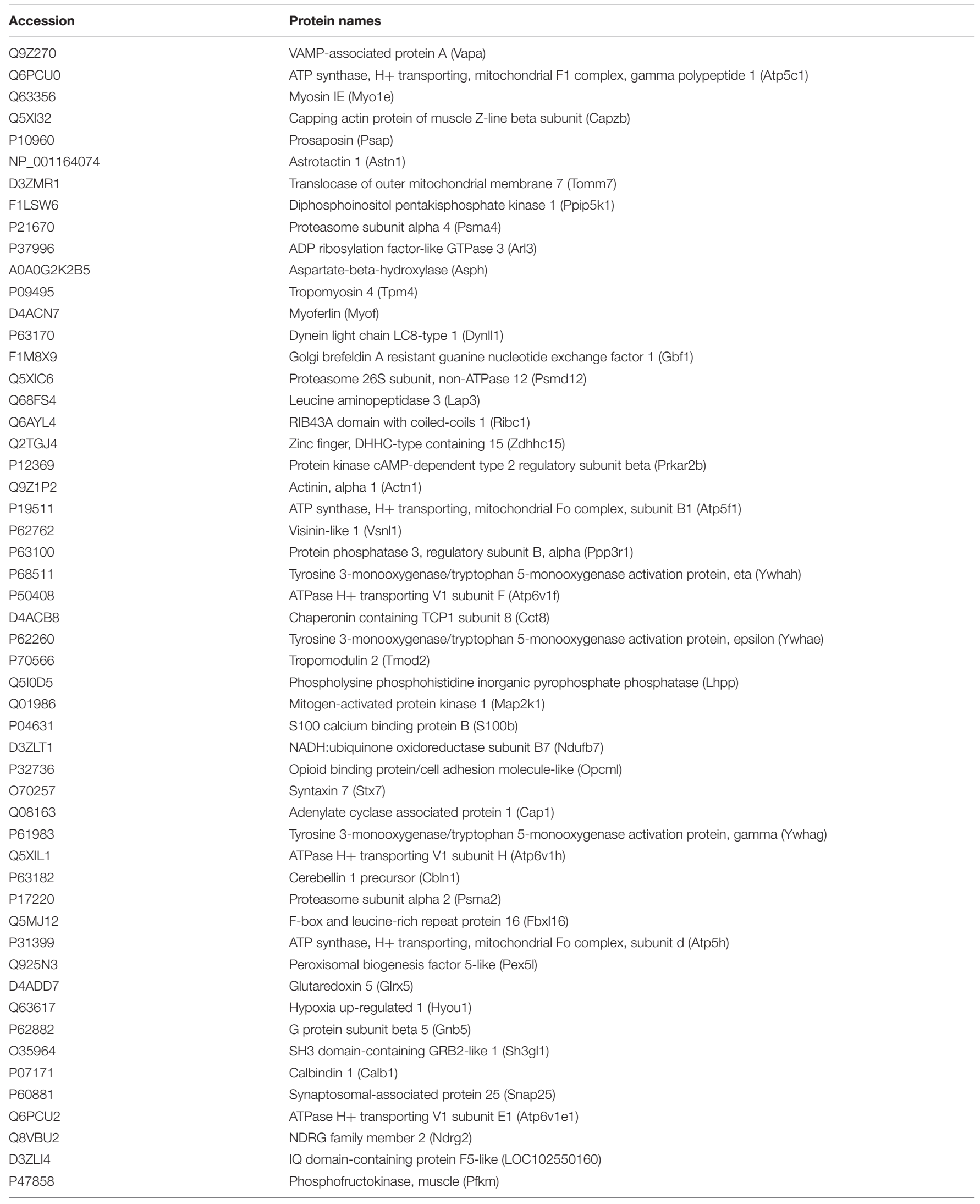


TABLE 2 | Continued

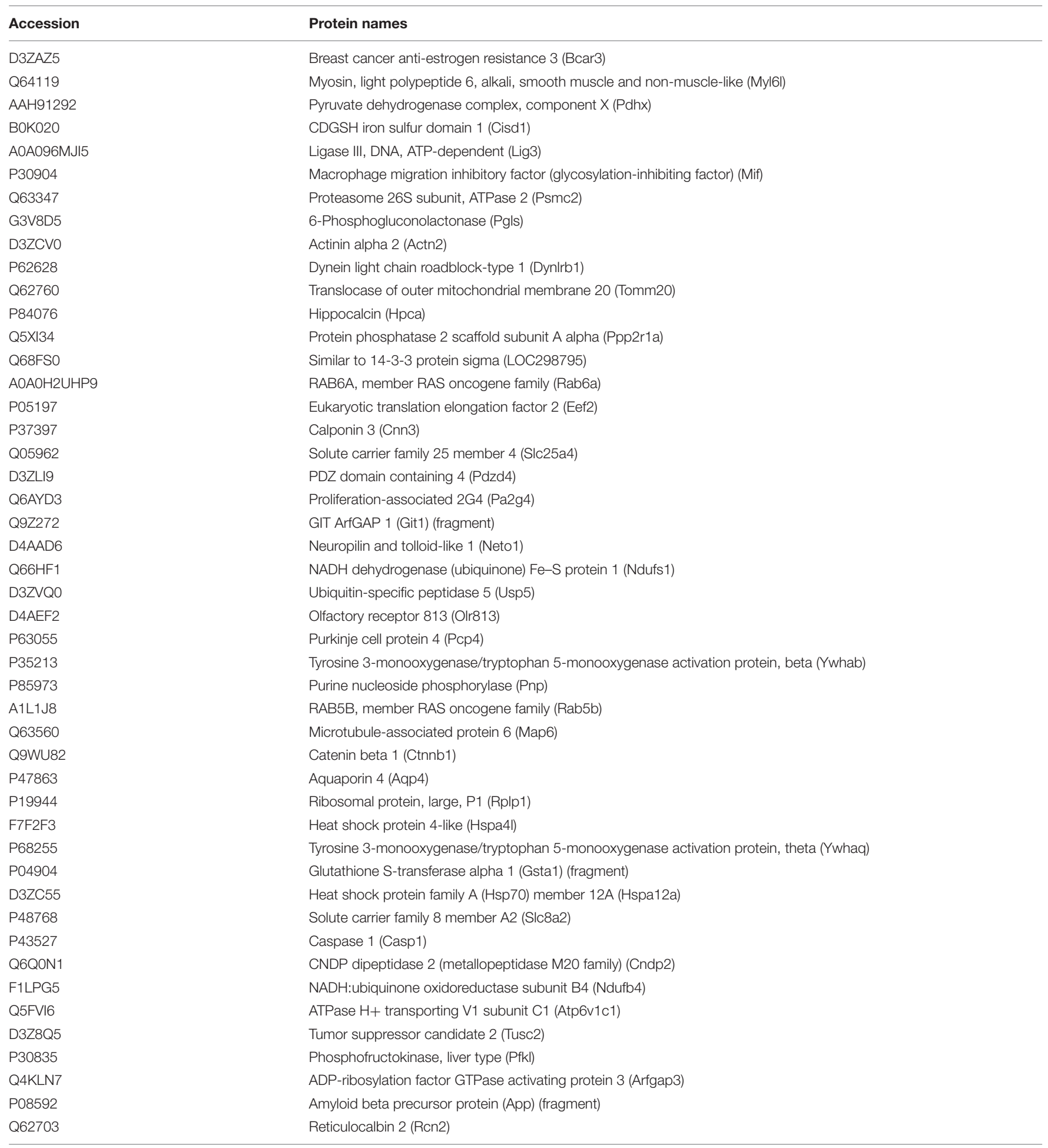

development, TST, OFT, and MWM in the offspring indicate that fear stress in pregnant rats could lead to developmental retardation and impairment of spatial learning and memory of offspring. In the MWM Test, not all the indexes between the two groups were significantly different, and the degree of impairment might not reach the disease state. However, it can be found that the learning and memory ability of OFSM rats was lower than that of ONC rats. These results indicate that offspring experienced an apparent state of behavioral change and growth retardation. 

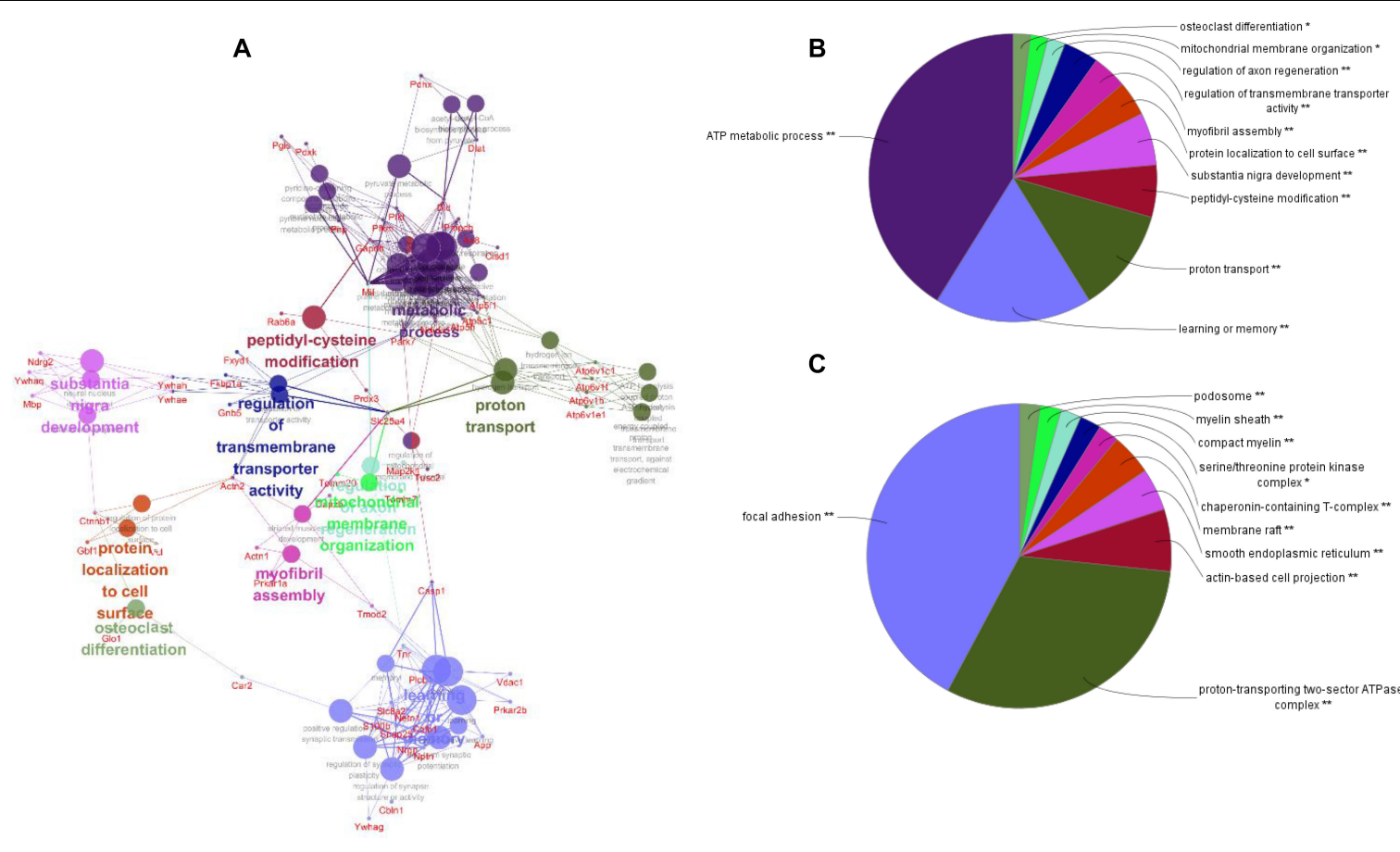

C

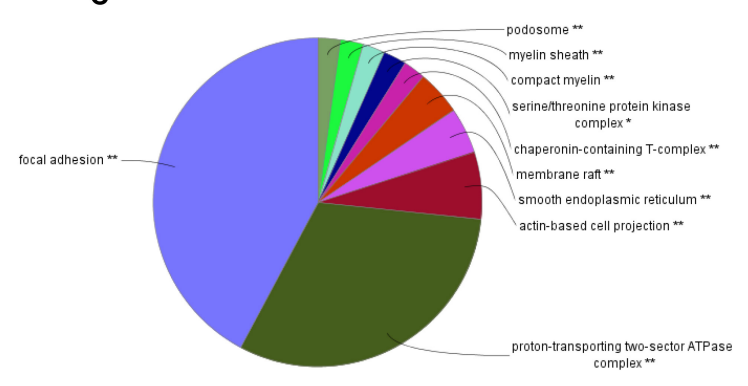

FIGURE 5 | The network of biological processes for the differentially expressed proteins (DEPs) (A). The biological processes [Gene Ontology (GO) category] of the DEPs were visualized using ClueGO software ( $p \leq 0.05$, kappa $\geq 0.4)$, and only the most significant interactions are shown. Each node represents a biological process, and the size of each node reflects the enrichment significance of the GO terms. The edges represent connections between the nodes. The color of the node represents the class. Mixed coloring represents multiple classes. Ungrouped terms are not shown. The distribution of DEPs according to biological process (B) and cellular location (C) was determined by ClueGO $(p \leq 0.05$, kappa $\geq 0.4)$, and only the most significant terms are shown. ${ }^{*} P<0.05$, ${ }^{\star \star} P<0.01$.

The hippocampus is thought to play an important role in the formation of early memories (Martin et al., 2008). Therefore, the hippocampal protein profiles of offspring were analyzed to identify potential biological relationships between prenatal stress and pathological changes in offspring. Using iTRAQ-based proteomics analyses, 158 proteins were found to exhibit a significant differential expression between the two groups. Among these, 58 were upregulated (Table 1), and 100 were downregulated (Table 2) in the hippocampus of the rats in the OFSM group relative to the ONC group, and the number of downregulated proteins was much bigger than that of the upregulated proteins. To develop the understanding of the effects of prenatal stress on the learning and memory behavior of the offspring, GO classification and pathway enrichment of the DEPs were performed. The pathway analyses showed that the DEPs mainly had functions in oxidative phosphorylation (00190), Alzheimer's disease (05010), amoebiasis (05146), and glycolysis/gluconeogenesis (00010) (Figure 6). The GO annotations also showed that the DEPs were mainly enriched in ATP metabolism and learning or memory. These results show that the expression of many learning- and memory-related proteins was changed. Meanwhile, the DEPs related to neurodegenerative diseases and learning or memories were mainly distributed in downregulated proteins (Table 2). These results suggest that fear stress in pregnant rats might increase the risk for neurodegenerative diseases in offspring.
In addition, the pathway network of the DEPs (Figure 6A) showed that the impairment of the hippocampal cGMP-PKG signaling pathway (04022), glycolysis/gluconeogenesis (00010), and the oxidative phosphorylation pathway (00190) may be the main reason for the learning and memory impairment in the offspring. The Alzheimer's disease pathway connected with the cGMP-PKG signaling pathway, oxidative phosphorylation (00190), and glycolysis/gluconeogenesis through four DEPs, five DEPs, and one DEP, respectively. These results lead us to speculate that the impairment of learning and memory in offspring was due to the disturbance of the cGMP-PKG signaling pathway and of oxidative phosphorylation in the hippocampus. Previous studies have found that cGMP signaling dysfunction is highly associated with memory deficit. Phosphodiesterase-5 inhibitors prevent the breakdown of cGMP and then stimulate cAMP/protein kinase A/cAMP element-binding protein (CREB) phosphorylation and $C G M P / P K G / C R E B$ pathway activation to enhance synaptic transmission (Sierksma et al., 2013; Zuccarello et al., 2020). In addition, the NO-cGMP-PKG pathway is able to regulate the production of neurotransmitters, including glutamate, to mediate synaptic plasticity, which is essential for learning and memory (Garthwaite, 2008). In addition, the NOcGMP-PKG signaling pathway regulates synaptic plasticity and fear memory consolidation by activating ERK/MAP kinase in the lateral amygdala (Ota et al., 2008). Furthermore, the CREB protein/CREB signaling pathway regulates the expression of 

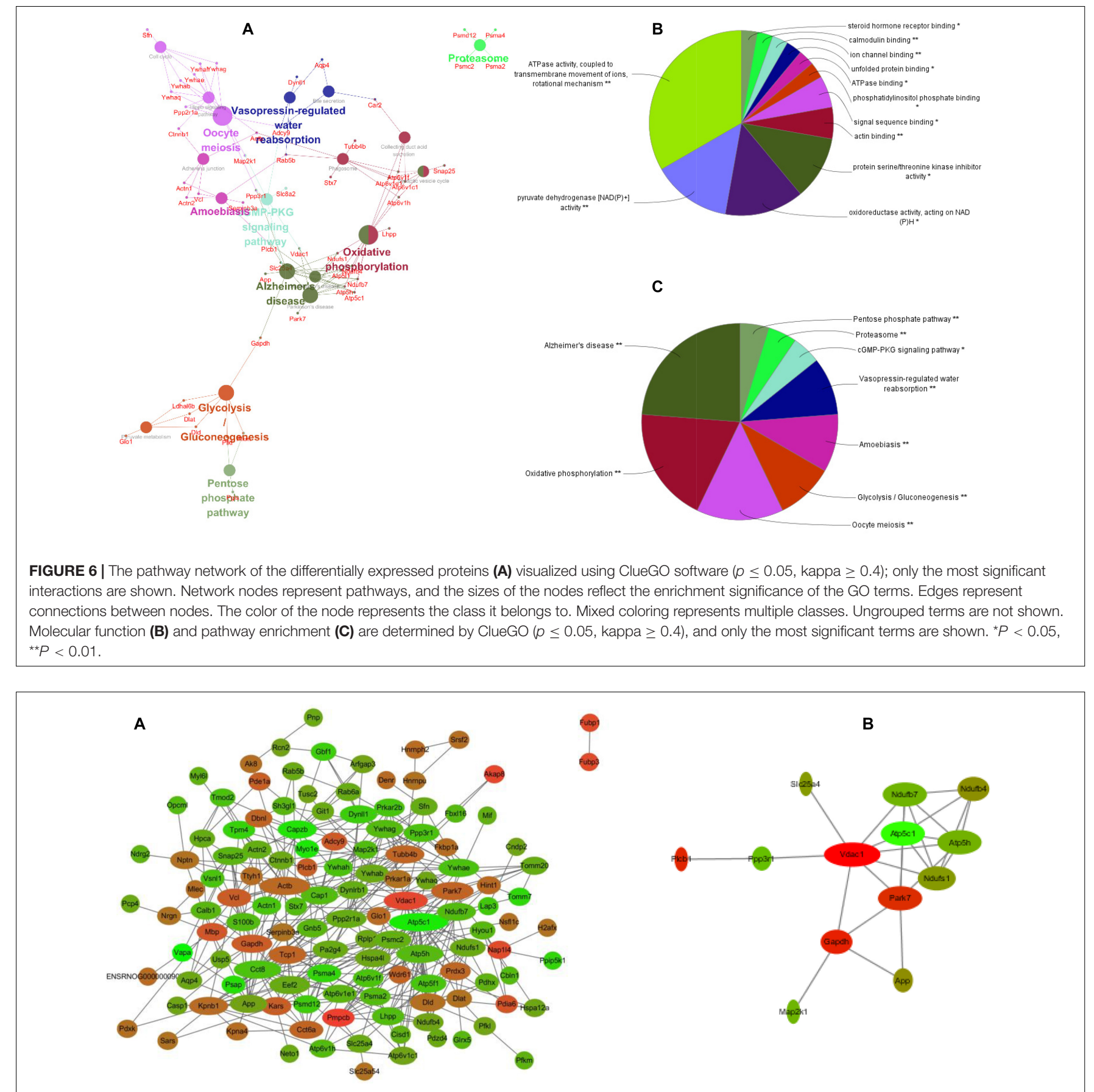

FIGURE 7 | The interaction network of differentially expressed proteins (DEPS) (A) and the interaction network of DEPs related to learning or memory and cyclic guanosine monophosphate-PKG pathway (B). Network nodes represent proteins; edges represent protein-protein associations. Red, significantly increased; green, significantly decreased; width, degree.

genes that are important for synaptic plasticity (Lonze and Ginty, 2002). Therefore, we speculated that fear stress during pregnancy might contribute to learning and memory impairment in offspring, and this might be associated with the dysfunction of the cGMP/PKG signaling pathway in the hippocampus of the offspring. Further analyses of the DEPs led us to conclude that most of those in the signaling pathway tended to decrease, such as Ppp3r1, Slc25a4, Map2k1, and Slc8a2. These results indicate that fear stress in pregnant rats may downregulate the cGMPPKG signaling pathway in the hippocampus of the offspring. The interaction network of DEPs related to learning or memory and to the cGMP-PKG pathway is shown in Figure 7B. To 
A

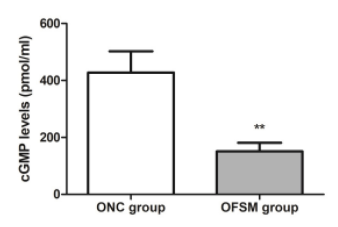

B

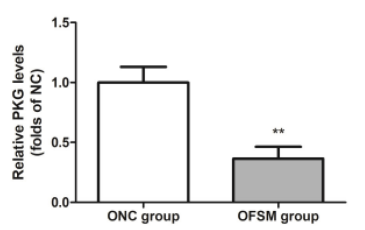

C

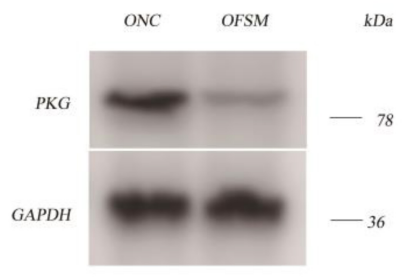

E

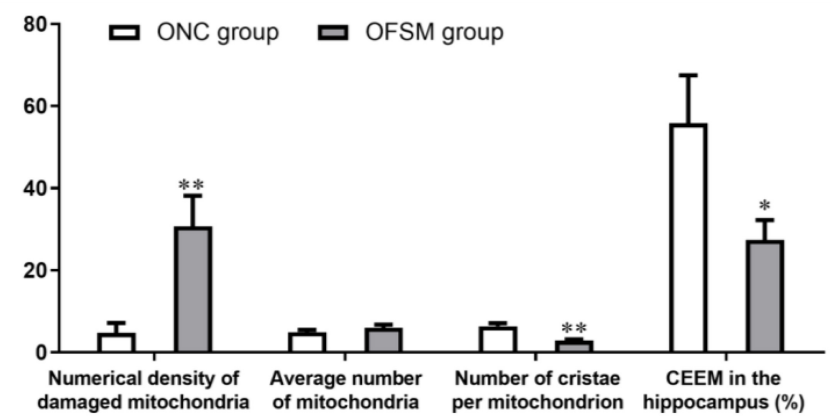

D ONC group OFSM group

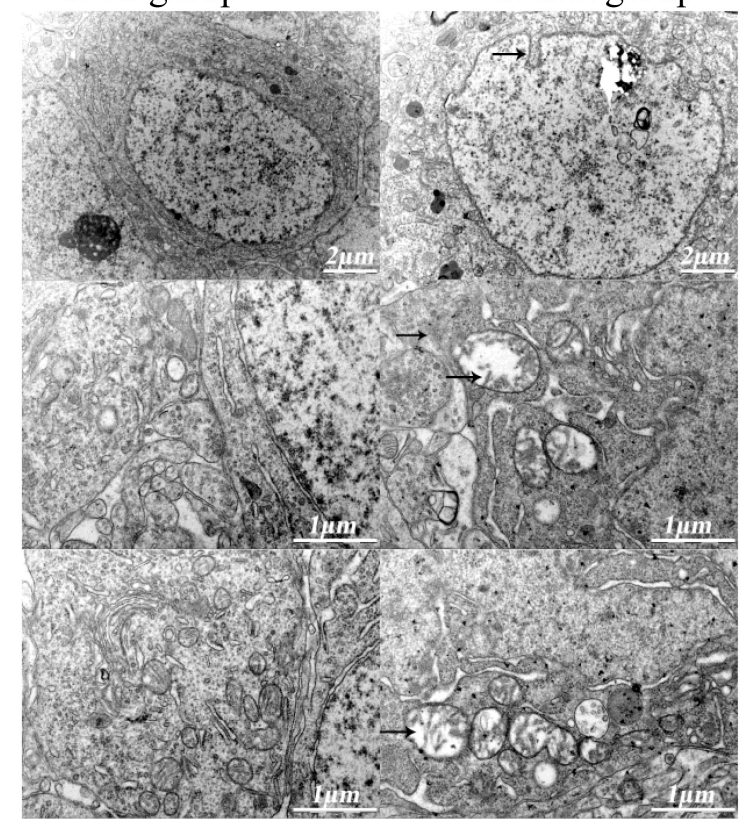

$\mathbf{F}$

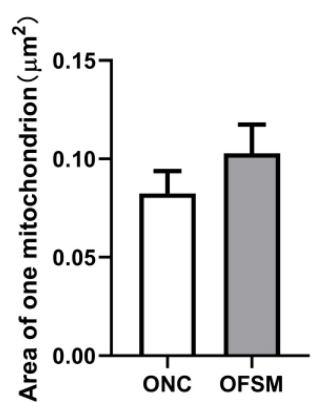

FIGURE 8 | The levels of cyclic guanosine monophosphate (cGMP) and protein kinase G (PKG) expression in the hippocampus of rats were detected by ELISA and western blotting assay, and the ultrastructure of the neurons was observed by electron microscopy. (A) Levels of cGMP. (B) PKG protein expression determined by western blotting. (C) Representative western blotting image of PKG; the results are presented as means $\pm \mathrm{SD}(n=6)$. ${ }^{\star \star} P<0.01$ versus offspring from the normal control group. (D) Representative electron microscopy pictures of hippocampal neurons (the ultrastructural changes are indicated by a black arrow; $n=4$ for each group). (E) Morphometric analysis of structural changes in the mitochondria of the hippocampus; the results are presented as means \pm SEM.

Magnification $\times 50,000$. (F) The percent numerical density of damaged mitochondria in the hippocampus; the results are presented as means \pm SEM. Magnification $\times 50,000 .{ }^{*} P<0.05,{ }^{\star \star} P<0.01$ versus offspring from the normal control group.

prove the scientific reliability of the hypothesis, the cGMP level and the expression of the PKG protein in the hippocampus of the offspring were detected by ELISA and western blotting, respectively. We found that the cGMP level and the expression of PKG protein decreased significantly in the hippocampus of the rats in the OFSM group. These results support our hypothesis. In addition, our previous experience has demonstrated that the phosphorylation of the CREB protein and the expression of Synapsin-1 in the hippocampus of the offspring significantly decrease in the maternal FSM group $(P<0.05)$ (Liping et al., 2013). These results suggest that fear stress during pregnancy inhibits the cGMP/PKG signaling pathway and leads to memory impairment in the offspring.
Furthermore, in the current study, the ultrastructures of the hippocampal neuron of the offspring were observed using electron microscopy. We observed that the synaptic structure was abnormal, the membranes of pre-axoaxonic synapses and post-axoaxonic synapses were not clear, the synaptic space disappeared, and the number of synapse vesicles was decreased. In addition, the neuronal mitochondria appeared swollen and vacuolated and their cristae were fragmented in the OFSM group. Synapse loss is observed in the early stages of Alzheimer's disease. Synapses are sites that demand large amounts of energy and depend on high levels of adenosine triphosphate derived from the mitochondria (Tang et al., 2019). Mitochondria 
within synaptic structures play an important role in maintaining functional neurotransmission, and this critical biological process is regulated by energy metabolism, mitochondrial distribution, mitochondrial transport, and cellular synaptic calcium flux (Tang et al., 2019). Thus, these ultrastructural changes could influence the energy metabolism of neurons and cause the impairment of learning and memory. According to GO classification, a high number of DEPs were associated with the oxidative phosphorylation pathway. These oxidative phosphorylation disorders in hippocampal neurons could influence the ability to remember and learn. These results suggest that fear stress in pregnant rats might affect the brain energy metabolism of their offspring via damage of neuronal ultrastructure, thereby increasing the risk for neurodegenerative diseases in offspring. In conclusion, we used LC-MS-based metabolomic and iTRAQ-based proteomic approaches to determine whether fear stress in pregnant rats affects the hippocampal metabonomics of their offspring. We found evidence that it can destroy the spatial learning and memory abilities of offspring. The mechanisms include affecting the brain oxidative phosphorylation process and inhibiting the cGMP-PKG signaling pathway.

\section{DATA AVAILABILITY STATEMENT}

All datasets presented in this study are included in the article/supplementary material.

\section{REFERENCES}

Abe, H., Hidaka, N., Kawagoe, C., Odagiri, K., Watanabe, Y., Ikeda, T., et al. (2007). Prenatal psychological stress causes higher emotionality, depressionlike behavior, and elevated activity in the hypothalamo-pituitary-adrenal axis. Neurosci. Res. 59, 145-151. doi: 10.1016/j.neures.2007.06.1465

Bindea, G., Mlecnik, B., Hackl, H., Charoentong, P., Tosolini, M., Kirilovsky, A., et al. (2009). ClueGO: a Cytoscape plug-in to decipher functionally grouped gene ontology and pathway annotation networks. Bioinformatics 25, 1091-1093. doi: 10.1093/bioinformatics/btp101

Chen, J., Woodbury, M. R., Alcorn, J., and Honaramooz, A. (2012). Dietary supplementation of female rats with elk velvet antler improves physical and neurological development of offspring. Evid. Based Complement. Alternat. Med. 2012:640680.

Chen, X., Zhu, Y., Wang, Z., Zhu, H., Pan, Q., Su, S., et al. (2016). mTORC1 alters the expression of glycolytic genes by regulating KPNA2 abundances. J. Proteomics 136, 13-24. doi: 10.1016/j.jprot.2016.01.021

Chiavegatto, S., Oliveira, C. A., and Bernardi, M. M. (1997). Prenatal exposure of rats to diphenhydramine: effects on physical development, open field, and gonadal hormone levels in adults. Neurotoxicol. Teratol. 19, 511-516. doi: 10.1016/s0892-0362(97)00045-7

Clark, J. D., Gebhart, G. F., Gonder, J. C., Keeling, M. E., and Kohn, D. F. (1997). Special report: the 1996 guide for the care and use of laboratory animals. ILAR J. 38, 41-48.

Doherty, F. D., O’Mahony, S. M., Peterson, V. L., O’Sullivan, O., Crispie, F., Cotter, P. D., et al. (2018). Post-weaning social isolation of rats leads to long-term disruption of the gut microbiota-immune-brain axis. Brain Behav. Immun. 68, 261-273. doi: 10.1016/j.bbi.2017.10.024

Garthwaite, J. (2008). Concepts of neural nitric oxide-mediated transmission. Eur. J. Neurosci. 27, 2783-2802. doi: 10.1111/j.1460-9568.2008.06285.x

Geng, S., Yang, L., Cheng, F., Zhang, Z., Li, J., Liu, W., et al. (2019). Gut microbiota are associated with psychological stress-induced defections in intestinal and blood-brain barriers. Front. Microbiol. 10:3067. doi: 10.3389/fmicb.2019.03067

\section{ETHICS STATEMENT}

The animal study was reviewed and approved by the Ethics Committee of Henan University of Chinese Medicine (Henan, China).

\section{AUTHOR CONTRIBUTIONS}

LY and YL conceived the study. JH, LC, and QW conducted the experiments. YL and JZ analyzed and interpreted the data. YL drafted the manuscript. X-ML and $\mathrm{PZ}$ revised the manuscript. YL, LY, X-HL, and ZS prepared the figures and the supplementary materials. All authors approved the final version of the manuscript.

\section{FUNDING}

This work was supported by the National Natural Science Foundation of China (grant nos. 81973596 and 81373790).

\section{ACKNOWLEDGMENTS}

We thank the Beijing Huada Protein Innovation R\&D Center Co., Ltd., for providing technical support, Cai-li Zhang and Ning Sun for providing electron microscope technical support, and Feng Cheng for providing drawing technical support.

Guan, S. Z., Ning, L., Tao, N., Lian, Y. L., Liu, J. W., and Ng, T. B. (2016). Effects of maternal stress during pregnancy on learning and memory via hippocampal BDNF, Arc (Arg3.1) expression in offspring. Environ. Toxicol. Pharmacol. 46, 158-167. doi: 10.1016/j.etap.2016.04.012

Haleem, D. J., Haque, Z., Ikram, H., and Haleem, M. A. (2014). Leptin and other hormonal responses to different stressors: relationship with stress-induced behavioral deficits. Pakistan Vet. J. 34, 504-507.

Ivani, S., Karimi, I., Tabatabaei, S. R., and Syedmoradi, L. (2016). Effects of prenatal exposure to single-wall carbon nanotubes on reproductive performance and neurodevelopment in mice. Toxicol. Ind. Health 32, 1293-1301. doi: 10.1177/ 0748233714555388

Jensen Pena, C., Monk, C., and Champagne, F. A. (2012). Epigenetic effects of prenatal stress on 11beta-hydroxysteroid dehydrogenase-2 in the placenta and fetal brain. PLoS One 7:e39791. doi: 10.1371/journal.pone.003 9791

Junlin, H., Liping, Y., Haijiao, W., Xinmin, L., Lei, C., Xianghong, Z., et al. (2018). Effects of fear impaired pregnant rats on plasma ACTH and GC content and development of offspring rats. CJTCMP 04, 1537-1539.

Katsura, M., Mohri, Y., Shuto, K., Tsujimura, A., Ukai, M., and Ohkuma, S. (2002). Psychological stress, but not physical stress, causes increase in diazepam binding inhibitor (DBI) mRNA expression in mouse brains. Mol. Brain Res. 104, 103-109. doi: 10.1016/s0169-328x(02)00219-x

Ladd, C. O., Huot, R. L., Thrivikraman, K. V., Nemeroff, C. B., Meaney, M. J., and Plotsky, P. M. (2000). Long-term behavioral and neuroendocrine adaptations to adverse early experience. Prog. Brain Res. 122, 81-103. doi: 10.1016/s00796123(08)62132-9

Laplante, D. P., Barr, R. G., Brunet, A., Galbaud du Fort, G., Meaney, M. L., Saucier, J. F., et al. (2004). Stress during pregnancy affects general intellectual and language functioning in human toddlers. Pediatr. Res. 56, 400-410. doi: 10.1203/01.pdr.0000136281.34035.44

Liping, Y., Gai, L., Haijiao, W., Jianghui, Z., Xinmin, L., Junlin, H., et al. (2018). Effects of terror stress of the pregnant rats on emotional changes of 21-day-old neonatal offspring. CJTCMP 01, 62-64. 
Liping, Y., Xinmin, L., Funing, Y., Lei, C., Xianghong, Z., and SIqing, C. (2013). The effect of chronic fear stress during pregnancy on the synaptic structure of neurons and the expression of p-CREB and SYN-1 in the hippocampal CA3 region of offspring rats. Lishizhen Med. Mater. Med. Res. 10, 2567-2568.

Liu, H., Lü, Z., Tian, C., Ouyang, W., Xiong, Y., You, Y., et al. (2019). [Mechanism of Shenbing decoction ? in the treatment of proteinuria in chronic kidney disease: a network pharmacology-based study]. J. South. Med. Univ. 39, 227234.

Lonze, B. E., and Ginty, D. D. (2002). Function and regulation of CREB family transcription factors in the nervous system. Neuron 35, 605-623. doi: 10.1016/ s0896-6273(02)00828-0

Martin, B., Brenneman, R., Becker, K. G., Gucek, M., Cole, R. N., and Maudsley, S. (2008). iTRAQ analysis of complex proteome alterations in 3xTgAD Alzheimer's mice: understanding the interface between physiology and disease. PLoS One 3:e2750. doi: 10.1371/journal.pone.0002750

Notarangelo, F. M., and Schwarcz, R. (2016). Restraint stress during pregnancy rapidly raises kynurenic acid levels in mouse placenta and fetal brain. Dev. Neurosci. 38, 458-468. doi: 10.1159/000455228

Ota, K. T., Pierre, V. J., Ploski, J. E., Queen, K., and Schafe, G. E. (2008). The NOcGMP-PKG signaling pathway regulates synaptic plasticity and fear memory consolidation in the lateral amygdala via activation of ERK/MAP kinase. Learn. Mem. 15, 792-805. doi: 10.1101/lm.1114808

Palmfeldt, J., Henningsen, K., Eriksen, S. A., Muller, H. K., and Wiborg, O. (2016). Protein biomarkers of susceptibility and resilience to stress in a rat model of depression. Mol. Cell. Neurosci. 74, 87-95. doi: 10.1016/j.mcn.2016.04.001

Partrick, K. A., Chassaing, B., Beach, L. Q., McCann, K. E., Gewirtz, A. T., and Huhman, K. L. (2018). Acute and repeated exposure to social stress reduces gut microbiota diversity in Syrian hamsters. Behav. Brain Res. 345, 39-48. doi: 10.1016/j.bbr.2018.02.005

Paukov, V. S., Kazanskaya, T. A., and Frolov, V. A. (1971). Quantitative analysis of some components of myocardial electron micrographs. Bull. Exp. Biol. Med. 71, 469-472. doi: 10.1007/bf00808503

Schmidt, M., Braun, K., Brandwein, C., Rossetti, A. C., Guara Ciurana, S., Riva, M. A., et al. (2018). Maternal stress during pregnancy induces depressive-like behavior only in female offspring and correlates to their hippocampal Avp and Oxt receptor expression. Behav. Brain Res. 353, 1-10. doi: 10.1016/j.bbr.2018. 06.027

Senko, T., Olexova, L., Mokosakova, M., and Krskova, L. (2017). Angiotensin II enhancement during pregnancy influences the emotionality of rat offspring (Rattus norvegicus) in adulthood. Potential use of the rat grimace scale. Neuro Endocrinol. Lett. 38, 117-123.

Sierksma, A. S., Rutten, K., Sydlik, S., Rostamian, S., Steinbusch, H. W., van den Hove, D. L., et al. (2013). Chronic phosphodiesterase type 2 inhibition improves memory in the APPswe/PS1dE9 mouse model of Alzheimer's disease. Neuropharmacology 64, 124-136. doi: 10.1016/j.neuropharm.2012.06.048

Smart, J. L., and Dobbing, J. (1971). Vulnerability of developing brain. II. Effects of early nutritional deprivation on reflex ontogeny and development of behaviour in the rat. Brain Res. 28, 85-95. doi: 10.1016/0006-8993(71)90526-9

Soliani, F. C. D. B. G., Cabbia, R., Kümpel, V. D., Batistela, M. F., Almeida, A. G., Yamauchi, L. Jr., et al. (2018). Unpredictable chronic prenatal stress and manifestation of generalized anxiety and panic in rat's offspring. Prog. Neuro Psychopharmacol. Biol. Psychiatry 85, 89-97. doi: 10.1016/j.pnpbp.2018. 03.005

Takeuchi, T., Iwanaga, M., and Harada, E. (2003). Possible regulatory mechanism of DHA-induced anti-stress reaction in rats. Brain Res. 964, 136-143. doi: 10.1016/s0006-8993(02)04113-6
Tang, J., Oliveros, A., and Jang, M. H. (2019). Dysfunctional mitochondrial bioenergetics and synaptic degeneration in Alzheimer disease. Int. Neurourol. J. 23, S5-S10.

Wang, T., Chen, H., Lv, K., Ji, G., Zhang, Y., Wang, Y., et al. (2017). iTRAQ-based proteomics analysis of hippocampus in spatial memory deficiency rats induced by simulated microgravity. J. Proteomics 160, 64-73. doi: 10.1016/j.jprot.2017. 03.013

Wang, X. F., Zhang, L., Wu, Q. H., Min, J. X., Ma, N., and Luo, L. C. (2015). Biological mechanisms of premature ovarian failure caused by psychological stress based on support vector regression. Int. J. Clin. Exp. Med. 8, 2139321399.

Wang, Y., Ma, Y., Cheng, W., Jiang, H., Zhang, X., Li, M., et al. (2015). Sexual differences in long-term effects of prenatal chronic mild stress on anxietylike behavior and stress-induced regional glutamate receptor expression in rat offspring. Int. J. Dev. Neurosci. 41, 80-91. doi: 10.1016/j.ijdevneu.2015.01.003

Weinstock, M. (2001). Alterations induced by gestational stress in brain morphology and behaviour of the offspring. Prog. Neurobiol. 65, 427-451. doi: 10.1016/s0301-0082(01)00018-1

Weinstock, M. (2017). Prenatal stressors in rodents: effects on behavior. Neurobiol. Stress 6, 3-13. doi: 10.1016/j.ynstr.2016.08.004

Welberg, L. A., and Seckl, J. R. (2001). Prenatal stress, glucocorticoids and the programming of the brain. J. Neuroendocrinol. 13, 113-128. doi: 10.1111/j. 1365-2826.2001.00601.x

Wu, S., Gao, Q., Zhao, P., Gao, Y., Xi, Y., Wang, X., et al. (2016). Sulforaphane produces antidepressant- and anxiolytic-like effects in adult mice. Behav. Brain Res. 301, 55-62. doi: 10.1016/j.bbr.2015.12.030

Xinmin, L., Liping, Y., Haijiao, W., Jianghui, Z., Junlin, H., and Xianghong, Z. (2017). Correlation between cognitive development and levels of dopamine and 3, 4-dihydroxyphenylacetic acid in the hippocampus in 80-day-old neonatal rats born of fear-impaired pregnant rats. Chinese J. Comp. Med. 11, 10-14.

Xu, M., Shi, J., Min, Z., Zhu, H., and Sun, W. (2019). A network pharmacology approach to uncover the molecular mechanisms of herbal formula kangbai-ling for treatment of vitiligo. Evid. Based Complement. Alternat. Med. 2019:3053458.

Youssef, N. A., Lockwood, L., Su, S., Hao, G., and Rutten, B. P. F. (2018). The effects of trauma, with or without PTSD, on the transgenerational DNA methylation alterations in human offsprings. Brain Sci. 8:83. doi: 10.3390/brainsci8050083

Zhang, Y., Yuan, S., Pu, J., Yang, L., Zhou, X., Liu, L., et al. (2018). Integrated metabolomics and proteomics analysis of hippocampus in a rat model of depression. Neuroscience 371, 207-220. doi: 10.1016/j.neuroscience.2017.12. 001

Zuccarello, E., Acquarone, E., Calcagno, E., Argyrousi, E. K., Deng, S. X., Landry, D. W., et al. (2020). Development of novel phosphodiesterase 5 inhibitors for the therapy of alzheimer's disease. Biochem. Pharmacol. 176:113818.

Conflict of Interest: The authors declare that the research was conducted in the absence of any commercial or financial relationships that could be construed as a potential conflict of interest.

Copyright (c) $2020 \mathrm{Li}$, Yang, Hou, Li, Chen, Zhu, Wang, Li, Zhao, Liu and Shi. This is an open-access article distributed under the terms of the Creative Commons Attribution License (CC BY). The use, distribution or reproduction in other forums is permitted, provided the original author(s) and the copyright owner(s) are credited and that the original publication in this journal is cited, in accordance with accepted academic practice. No use, distribution or reproduction is permitted which does not comply with these terms. 